\title{
Emerging Structural
}

Understanding of Amyloid Fibrils

by Solid-State NMR

\section{Review Article}

\section{Author(s):}

Meier, Beat H.; Riek, Roland; Böckmann, Anja

Publication date:

2017-10

Permanent link:

https://doi.org/10.3929/ethz-b-000193533

\section{Rights / license:}

Creative Commons Attribution-NonCommercial-NoDerivatives 4.0 International

Originally published in:

Trends in Biochemical Sciences 42(10), https://doi.org/10.1016/j.tibs.2017.08.001 


\title{
Emerging structural understanding of amyloid fibrils by solid state NMR
}

Beat H. Meier ${ }^{{ }^{*}}$, Roland Riek ${ }^{1 *}$, and Anja Böckmann ${ }^{2 *}$

${ }^{1}$ ETH Zürich, Physical Chemistry, Vladimir-Prelog-Weg 2, 8093 Zürich, Switzerland

${ }^{2}$ Institut de Biologie et Chimie des Protéines, Bases Moléculaires et Structurales des Systèmes Infectieux, Labex Ecofect, UMR 5086 CNRS, Université de Lyon, 7 passage du Vercors, 69367 Lyon, France

*Correspondence: a.bockmann@ibcp.fr(A. Böckmann), roland.riek@phys.chem.ethz.ch (Roland Riek), beme@ethz.ch (Beat H. Meier),

Keywords : amyloid fibrils, structure, NMR, amyloid- $\beta$

\begin{abstract}
Amyloid structures at atomic resolution remained elusive for a long time, which was mainly due to the extensive polymorphism they present, as well as to their polymer properties hampering structural studies by classical approaches. Progress in sample preparation, as well as solid-state NMR methods, recently enabled the determination of high-resolution 3D structures of fibrils, as for example amyloid- $\beta$ involved in Alzheimer's disease. Notably, the simultaneous but independent structure determination of A 1-42 by two independent laboratories with virtually identical results has highlighted how structures can be obtained on which further functional investigation can be based.
\end{abstract}


Amyloid (see glossary) fibrils are a product of self-assembly of proteins. Some of them are related to diseases, most prominently amyloid- $\boldsymbol{\beta}$ (see glossary) and tau to Alzheimer's disease (see glossary) \{Glenner:1984uw, Masters:1985wb\} but also play a role as functional amyloids \{Maji:2009hv\}. Their first design as soluble protein is to serve a determined function, which is not always known. Fibrils present interesting objects from a structural biology point of view. While it is well known that proteins can adopt different threedimensional (3D) structures in the context of their function by differences in the local environment such as $\mathrm{pH}$, salt, membranes or ligand binding, amyloidogenic peptides and proteins undergo a change in secondary, tertiary and quaternary structure through aggregation triggered only by a concentration change of the amyloid protein.

Resisting classical structure-determination approaches, such aggregates were long assumed to all adopt a generic fold consisting of two layers of inter-molecular $\beta$-sheets \{Dobson:2003el, Petkova:2002dr\} or variations like a serpentine \{Kajava:2004da\} with monomers stacking on top of each other to form the amyloid fibril. However, it has recently become apparent that protein fibrils sample a comparable conformational space as globular and membrane proteins, and include complex combinations of $\beta$-strands in the case of amyloids, and also $\alpha$-helices for fibrils formed in other contexts \{Sborgi:2015iv, Dvir:2009fu, TayebFligelman:2017kv\}. Structural insight into the fold of fibrils was delayed due to the nature of these objects. Protein fibrils do not normally show long-range order; they are often bent and flexible along their axis (see Figure 1), and resist thus to atomic resolution structural studies by x-ray diffraction. With the advent of solid-state NMR (see glossary) as a structural technique for (biological) solids, structures of protein fibrils at atomic resolution became accessible, and diverse folds of these objects were revealed. While solid-state NMR allows structural studies of large objects lacking long-range order, local order is still a prerequisite, and first studies by this technique were hampered by suboptimal sample preparations, as well as the inherent propensity of many fibrils to form structurally distinct polymorphs (see glossary), which are difficult to separate. The establishment of rigorous production and purification techniques, as well as seeding (see glossary) approaches, allowed recently to acquire high-resolution NMR spectra of protein fibrils adequate for the assignment of the NMR resonances. The measurement of structural restrains then allowed 
the determination of the 3D structures. We review here the recently determined amyloid structures with a focus on amyloid- $\beta$ fibrils involved in Alzheimer's disease.

\section{Amyloid structures have local order but limited long-range order}

Multimeric protein superstructures can result in highly ordered entities (certain virus capsids and envelopes, ferritins, bacterial needles etc.), but can also form less ordered assemblies, which for example bend and curve irregularly, as is the case for most protein fibrils. Protein fibrils indeed show a quasi one-dimensional growth, and if the fibrils can consist of several protofibrils, only few specific lateral interactions between entire fibrils exist, even if fibrils often bundle, but likely via non-specific contacts. These features prevent in most cases atomic-resolution structural studies by established techniques, as x-ray crystallography, and complicate electron microscopy, as the absence of long-range order (e.g. exact translational symmetry) prevents diffraction and electron-density reconstruction to be used with their full potential. Despite these elements of randomness, other types of long-range, like a constant cross section and a constant supertwist are often observable. It should also be noted that for peptides fibrils consisting of smaller peptides, crystalline objects could be grown, and their structure has been determined \{Sawaya:2007em\} and that quite high resolution cryoelectron microscopy on A $\beta 1-42$ has been obtained recently $\{$ Schmidt:2015df\}.

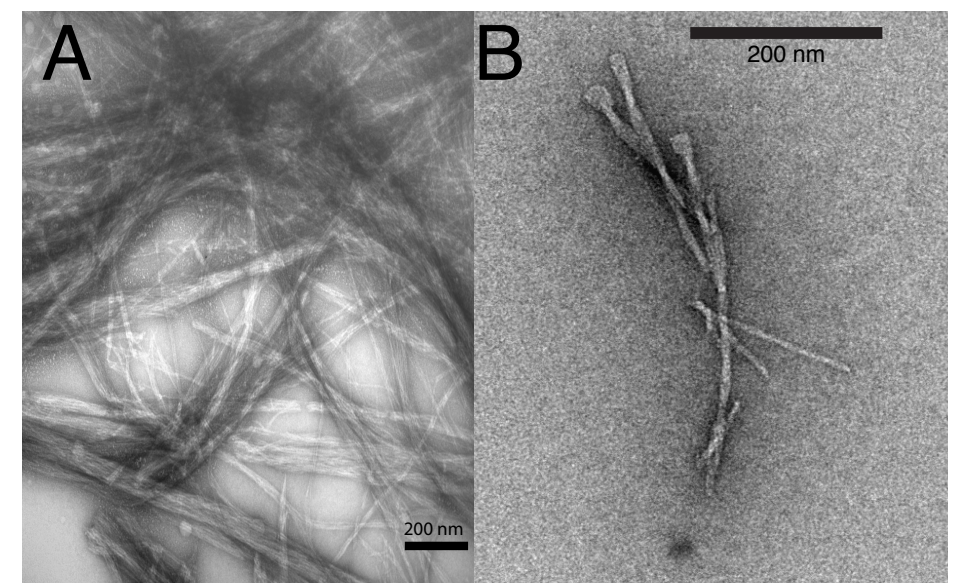

Figure 1: EM pictures (negative stain) of two different protein fibrils, HET-s(218-289) (A) and Aß1-42 (B), scale bars indicate $200 \mathrm{~nm}$.

The need of making samples with a single polymorph 
Besides the technical challenges introduced in the preceding paragraph, the ability to prepare samples showing a single polymorph presented a major breakthrough for structural studies. Polymorphism is pervasive in many fibrils, and leads typically to the observation of several signals per single residue in the solid-state NMR spectra because the conformations are distinct in the different polymorphs, and chemical shifts respond in a particularly sensitive manner to differences in dihedral angles. Exceptions can be fibrils with more than one symmetrically inequivalent molecules in the fibril \{Fitzpatrick:2013do\} where multiple peaks can be observed per atom even in the absence of polymorphism, or polymorphs with almost identical local conformation but a different supertwist \{Seuring:2017hg\} or quaternary structure \{Fitzpatrick:2013do\} that can not be distinguished by NMR because they have a close-to-identical local structure. Both exceptions are not applicable for the cases discussed here. In the presence of many polymorphs, the NMR spectra become therefore crowded and their analysis becomes difficult due to the strong overlap of signals. Still, for some fibrils, only a single polymorph has been observed under physiological conditions, as for example in HET-s(218-289) (see glossary) \{Wasmer:2008gc, vanMelckebeke:2010kz\}. In many other cases polymorphism is often observed \{Gath:2014ff, Walti:2016fn, Petkova:2005eh, Lu:2013bb\} and pure protein preparation and the tight control of fibrillization conditions or seeding techniques are key to obtain a homogeneous sample. In this context it is interesting to note that peptide preparations using synthesizers typically results in less homogeneous fibrils than from recombinant sources \{Finder:2010jw\} and that preparations from recombinant sources without any addition of amino acids to the native sequence are considered the most relevant. Also, reproducible conditions are central for NMR studies, as several different samples have to be prepared for structural studies $\{L u: 2013 b b$, vanMelckebeke:2010kz\}. Reproducibility is best guaranteed by tight control of the physico-chemical conditions including salt content, metal ions, buffer composition, temperature and $\mathrm{pH}$ or/and by seeding fresh material with a "mother" fibril sample.

Once a sample condition with a single polymorph is established, it is unclear whether the selected polymorph is relevant in the context of disease. A correlation between patientderived material and the NMR sample is therefore necessary by using conformation-specific antibodies \{Walti:2016fn\}, spectroscopic signatures \{Anonymous:2007ju\} or by showing biological activity such as transmissibility potential \{Tuttle:2016h/\}. Alternatively, seeding 
the stable isotope labeled monomeric material using brain homogenates from deceased patients may result in disease-relevant structural studies \{Lu:2013bb, Qiang:2017ek\}. Still their relevance remains difficult to establish since other forms could be formed during seeding and NMR sample preparation (see also below). Within the context of the various polymorphisms observed in vitro, it is interesting to note that also in vivo different strains and structural polymorphisms have been observed with distinct biological properties, e.g. different toxicity and transmissibility, and one may thus argue that the various polymorphs represent different strains of a disease \{Bousset:2013im, Qiang:2017ek, Prusiner:2012fp, Soto:2012hc\}.
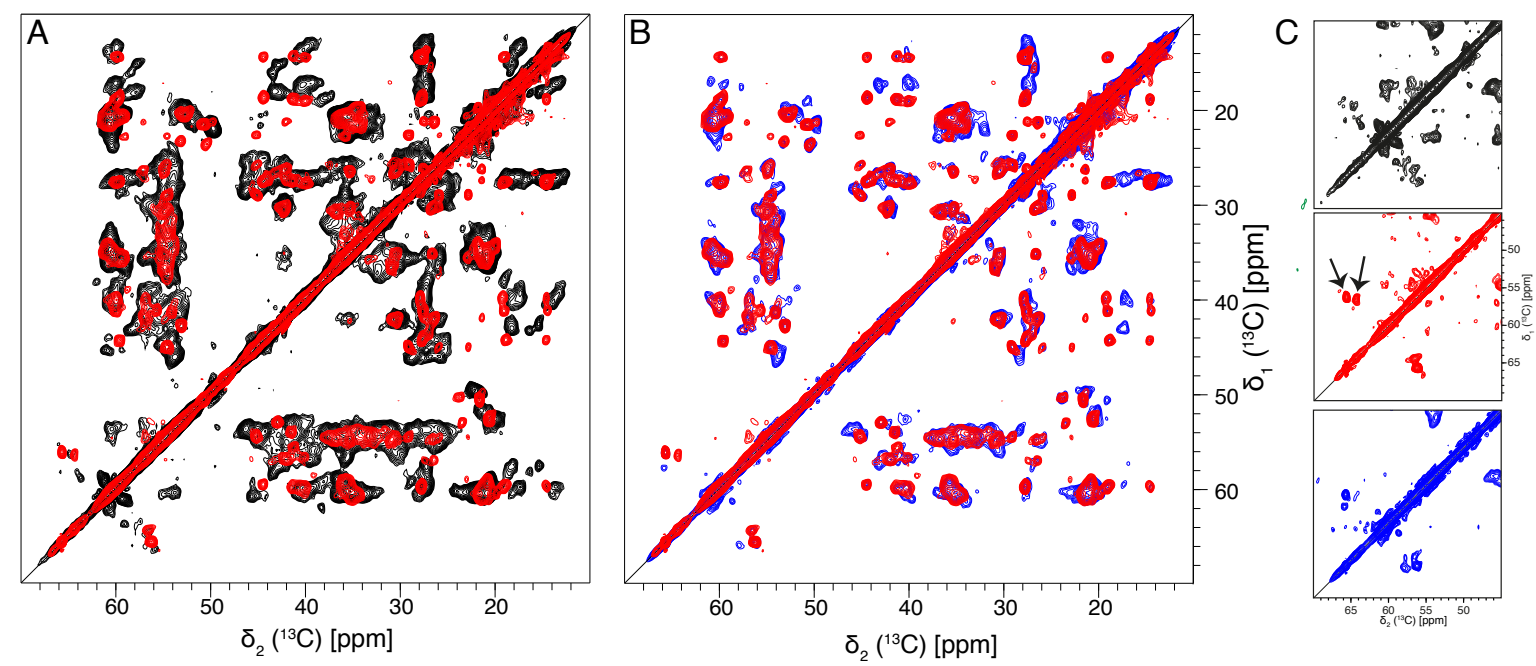

Figure 2: $\left[{ }^{13} \mathrm{C},{ }^{13} \mathrm{C}\right]$-correlation spectra of different polymorphic mixtures of $A \beta 1-42$ fibrils. The black spectrum in $(A)$ is from a sample which shows a broad distribution with at least 6 polymorphs, while the red spectrum (in $A$ and $B$ ) is from a sample with a single polymorph and the blue spectrum (shown in (B)) is from a sample with several polymorphs. The number of polymorphs can best be seen in the serine region of the spectra shown in (C) and are marked by an arrow in the red extract. This region shows a single cross peak between the ${ }^{13} C \alpha$ and the ${ }^{13} C \beta$ atoms for each serine residue. Since $A \beta 1-42$ has two serine residues only two cross peaks are expected as found for the red spectrum attributed to a fairly pure single polymorph ( 2 serines), while the blue sample contains the red polymorph and, in addition, a second one, and the black spectrum at least three polymorphs (i.e. $3^{*} 2$ serine 
cross peaks). For details of sample preparation see $\{$ Walti:2016fn\}. Figure adapted from \{Walti:2016fn\}.

\section{Solid-state NMR unblocked the situation}

The strength of solid-state NMR resides in the need of only local order, which can be high in protein fibrils. In contrast to $x$-ray and EM, NMR structure determination is a spectroscopic method based on combining short-distance information $(<12 \AA)$, and structures result from the triangulation using a large array of distances which arise from structural restraints detected in 2D correlation spectra $\{$ Wuthrich:2003wx\}. Solid-state NMR is a recent player in biophysics, and protocols needed to be established directly on fibril samples in order to address and maximally use the specific properties of this structural class of proteins \{Schutz:2015gi, Wasmer:2008gc\}. Advances in technology and methodology now enable the recording of spectra with high spectral resolution and thus resolved resonances of proteins ranging up to six hundred amino-acid residues in size (per monomer). Sequential assignments of the NMR resonances have been established early on \{McDermott:2000kx, Bockmann:2003vc, Pauli:2001vb\}, and can be achieved today for up to about 400 amino acid residues $\{$ Habenstein:2011cl\}. They allow directly to assess locally the secondary structure from the ${ }^{13} \mathrm{C} \alpha$ and ${ }^{13} \mathrm{C} \beta$ chemical shift (see glossary) via their difference with the random coil chemical shift \{Bax:1991th\}. First structure determination protocols for globular proteins, relying on distance measurements via the dipolar couplings (see glossary) present between the nuclei and which depend on the inverse 6 'th power of the internuclear distance, followed soon \{Castellani:2002th, Anonymous:2008cl, Loquet:2008ti\}. Typically carboncarbon, carbon-nitrogen and proton-proton distances can be measured. For the elucidation of the structures of amyloids it is in particular important to be able to distinguish between intermolecular and intramolecular distances. Essential information comes in this context from scanning transmission electron microscopy (STEM) mass-per-length (see glossary) where measurements allow to access the mass per length of the fibrils and thus to define the number of lateral neighbors. A further difficulty less present in globular proteins is the high proportion of intermolecular contacts, which have to be differentiated from intermolecular ones for successful structure calculation. For this, isotope labeling (see glossary) schemes, as well as a protocol for quantitative comparison of intermolecular and intramolecular crosspeaks in the NMR spectrum had to be established. Mixed only ${ }^{15} \mathrm{~N}-$ 
labeled and only ${ }^{13} \mathrm{C}$-labeled samples are suited to measure distances between nitrogen and carbon spins of different monomers. Such experiments also allow early-on to determine if the fibrils are formed by in-register parallel interactions between the different layers \{Schutz:2015gi\}. Since assignments of the cross-peak signals in solid-state NMR spectra are often highly ambiguous, due to still somewhat lower spectral resolution than in solutionstate NMR, structure calculation remains difficult when done using the established protocols from solution NMR \{Wuthrich:2003wx, Guntert:2002ut\}. Instead, a calculation based on exclusively unambiguous restraints is carried out first in order to determine the fold (see Figure 3). A full structure calculation is then followed using automated assignments of all cross signals \{Schutz:2015gi, Walti:2016fn\}.

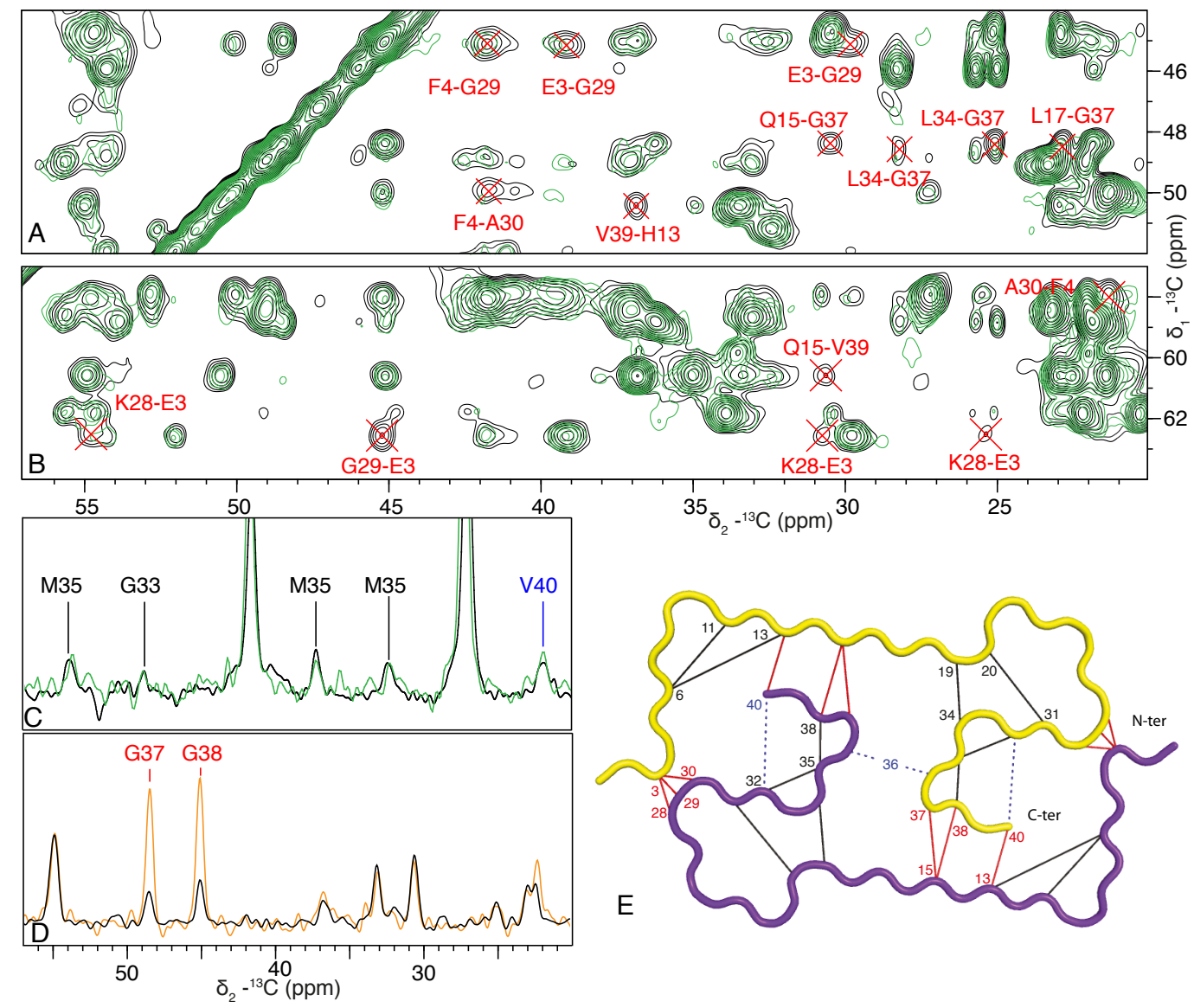

Figure 3: Osaka mutant $A \beta 1-40 E 22 \triangle$ spectra. $(A, B)$ Extracts from 2D PAR (see glossary) spectra recorded on fully labeled (black) and diluted (green) samples. Intermolecular cross peaks can be identified by the signal attenuation in the green spectra. (C) 1D PAR traces extracted at the 1 e32 $c \delta_{1}$-resonance at $14.8 \mathrm{ppm}$ where intramolecular crosspeaks retain 
full intensity. The signal labeled in blue denotes an ambiguous intramolecular peak not used in the calculation but confirmed by the structure. (D) 1D trace from PAIN (see glossary) spectrum from mixed ${ }^{15} \mathrm{~N}$ and ${ }^{13} \mathrm{C}$ sample (orange), compared to an NCA spectrum recorded on a fully labeled ${ }^{15} \mathrm{~N}$ and ${ }^{13} \mathrm{C}$ sample in black. The trace is taken at the $\mathrm{Q} 15 \mathrm{~N} \varepsilon 2$ frequency (108.86 ppm). Resonances where intermolecular cross peaks (orange) are much stronger are due to lateral intermolecular contacts (Q15-G37, Q15-G38), peaks with similar intensity may be contributions from the adjacent layer or ${ }^{13} \mathrm{C}$ natural abundance intramolecular contributions. Spectrally unambiguous peaks are labeled in (A-D), and are shown in (E) (with the color code red for intermolecular peaks, black for intramolecular and blue for ambiguous with respect to intra/inter) on the backbone structure of $A \beta 1-40 E 22 \Delta$ with two monomers in yellow and violet. Figure adapted from \{Schutz:2015gi\}.

The resulting 3D structures represented by a bundle of conformers then deliver an atomic picture of the protein fibrils (see Figure $4 \mathrm{C}$ ). In NMR, the resolution of the structure depends on the number of distance restraints (see glossary). In the $\mathrm{A} \beta$ structures discussed here, 500-1000 restraints were used. Not only the number but also the quality of the restrains is of importance. The more the restraints are distributed over the entire structure, the more reliable the structure will be. If structures can be solved with a lower number of restraints, it often remained unclear which parts were well defined without showing a representative bundle of structures. This has resulted in the past in models which approximate nature was difficult to assess to the outsider.

\section{Atomic-resolution 3D structures yield insight into fibril architecture}

The two structures of $A \beta 1-42$, as well as the structure of the Osaka mutant (see glossary) $A \beta 1-40 E 22 \Delta$ are shown in Figure 4. One can see that the structures are rather complex and distinct from the simple $\beta$-strand-turn- $\beta$-strand fold suggested initially. Both represent dimeric folds, in line with the mass-per-length determined by STEM, with the backbones of the Osaka mutant $A \beta 1-40 E 22 \triangle$ being highly intertwined, and $A \beta 1-42$ showing less intermolecular contacts. In both structures, the hydrophobic residues are mainly located in the inside of the protein, as also observed in globular proteins. The polar and charged residues point mainly to the outside. If charged residues point to the inside, their charge is compensated as for example in the salt bridges between residues $\mathrm{H6}, \mathrm{E} 11, \mathrm{H} 13$ and $\mathrm{V} 4 \mathrm{O}$ in 
the Osaka mutant $A \beta 1-40 E 22 \Delta$. In $A \beta 1-42$, no charged residues are found in the interior of the protein fibril. The most fundamental difference between the two structures lies in the attachment of the $\mathrm{N}$-terminal domain to the fibrillar core. Indeed, the $\mathrm{N}$-terminus is attached via a salt bridge between residues D3 and K28 in the Osaka mutant A $\beta 1-40 E 22 \Delta$, whereas in A $\beta 1-42$ it shows $\beta$-strand structure, but remains at least partially flexible, as no extensive network of restraints can be observed between the $\mathrm{N}$-terminus and the amyloid core in A 1 1-42. In both structures, glycine residues are used for turns, which allows the backbone to easily adopt a kringel- or horseshoe-like structure. In both constructs, V36 represents the center of the structure and is heavily involved in intermolecular contacts. We have speculated with respect to early disease onset that the familial mutations in the loop around residue E22 result in an exposure or not of $\mathrm{K} 28$ to the outside, so it can interact with the $\mathrm{N}$-terminal residues $\{$ Schledorn:2015ds\}. This attachment of the $\mathrm{N}$-terminal may present a hallmark for early-onset, but these hypotheses remain to be explored experimentally. In $A \beta 1-42, K 28$ is indeed also oriented toward the exterior of the fibril, but is interacting with the carboxy-terminal of A42. It will be interesting to investigate the Osaka mutant E22 $\Delta$ of Aß1-42.

An important success of the solid-state NMR method was that a second, independent NMR structure determination carried out in parallel for essentially the same polymorph of AB1-42 (as revealed by highly similar chemical shifts \{Ravotti:2016fv\}) yielded a highly similar structure (albeit the construct used has a methionine residue at position $0\{W a l s h: 2009 \mathrm{~kg}\}$ ). The small differences in the structure and chemical shifts observed might be attributed to this additional methionine residue. 

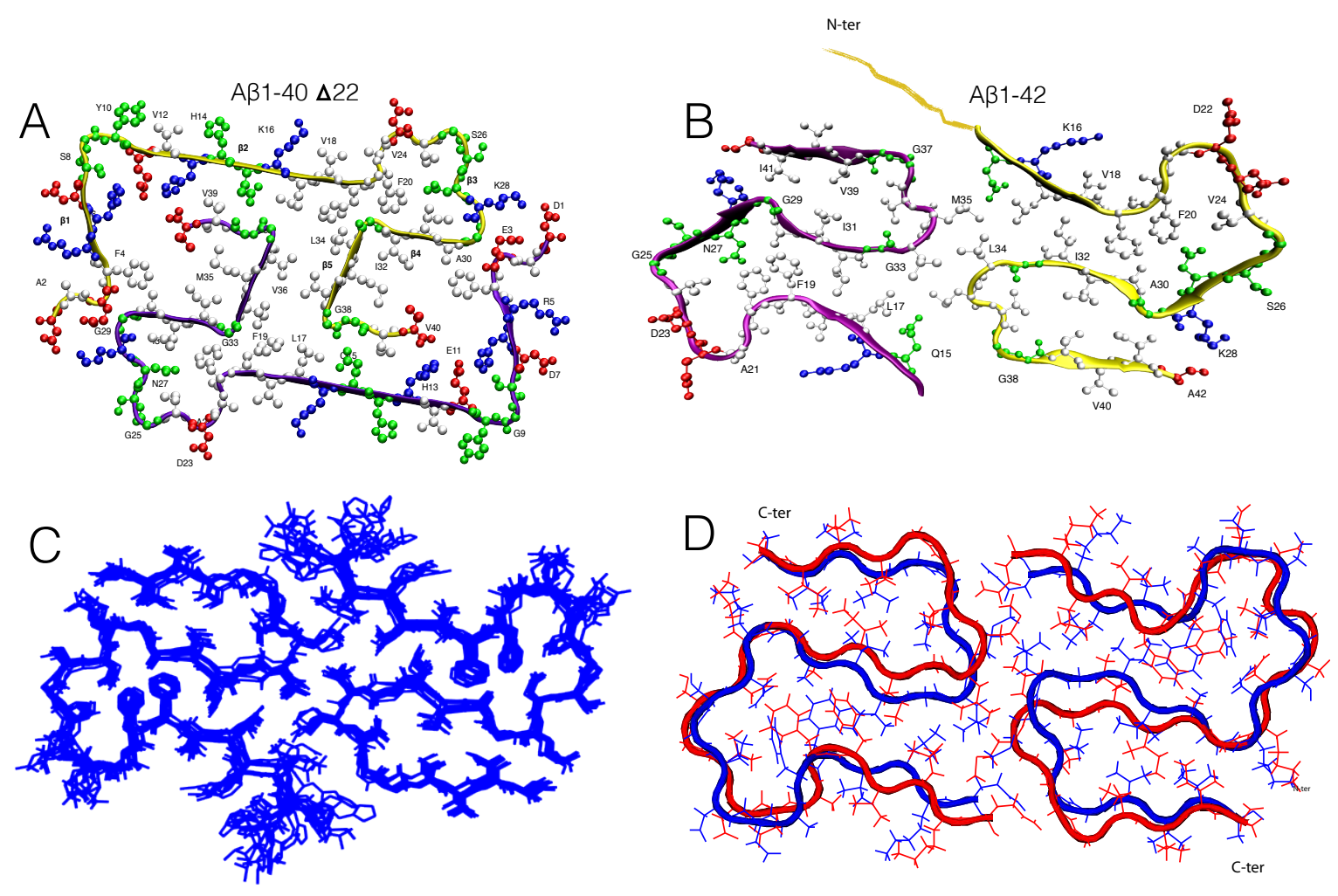

Figure 4: Structures of amyloid- $\beta$ : (A) Osaka mutant $A \beta 1-40 E 22 \Delta$, view perpendicular to fiber direction, white balls denote hydrophobic residues, green balls polar (including glycine), red and blue balls negatively and positively charged residues, respectively, (B) A $\beta$ 1-42 from the "red" polymorph of Fig. 2 (from ref. \{Walti:2016fn\}); only residues 15-42 are shown (in yellow the $\mathrm{N}$-terminal tail is added on one monomer). (C) Bundle of 10 best conformers of (B) of residues 15-42, (D) Overlay of the structures obtained by Wälti et al. \{Walti:2016fn\} (blue) and Colvin et al. \{Colvin:2016jx\} (red) from samples formed under similar conditions showing a very similar chemical-shift fingerprint. The sample used by Colvin contains an additional methionine residue at the $\mathrm{N}$-terminal end of the amino-acid sequence (residue 0 ).

\section{Interactions with amyloid dyes}

The available structures of amyloid fibrils open an avenue towards the study of amyloidmarker interactions and their structure-based rational design. Recent solid-state NMR studies (both by ligand-induced chemical shift perturbations as well as ligand-amyloid intermolecular polarization transfers) identified the binding mode of the well known amyloid dye 
Congo red with the functional prion HET-s(218-289) amyloid \{Schutz:2011kw\}. This work revealed that Congo red binds along the fibril axis with the two negative charges located on the Congo red $\mathrm{SO}_{3}{ }^{-}$groups (which are spaced by $19 \AA$ ) interacting with positively charged lysines, which match exactly the corresponding distance in every second layer of the fibril. Solid-state NMR has also been used to study another class of amyloid interacting molecules, luminescent conjugated polymers \{Herrmann:2015en\}. These are compounds which show regularly spaced negatively charged groups, $\mathrm{COO}^{-}$, which are attached to a polythiophene backbone. Studies have shown that these compounds bind in a similar manner to amyloids as Congo red; indeed, the negative charges bind to the same lysine residue in the model mutant HET-s(218-289) E265K amyloid, which now contains a lysine ladder as depicted in Figure 5 \{Herrmann:2015en\}. Using the ligand-induced chemical-shift perturbations in combination with ligand-amyloid polarization transfers the structure of the complex was modeled and the binding mode revealed (Fig. 5). The spacing of the negative charges for different polythiophenes depends on the polymer backbone, and can be adapted. For compounds where the negative charges are spaced by $9 \AA$, the binding is observed for every monomer of HET-s which forms two layers in this amyloid. Polythiophenes with a spacing of 4.5 ̊̊ have been designed as well, and can bind to sites where every layer of the amyloid displays the same positive charge. Charge alone, however, does not seem sufficient for a high binding affinity; a groove running along the fibril in HET-s(218-289) near the Lys binding site seems to play a role in interactions mediated via the neighboring backbone atoms.

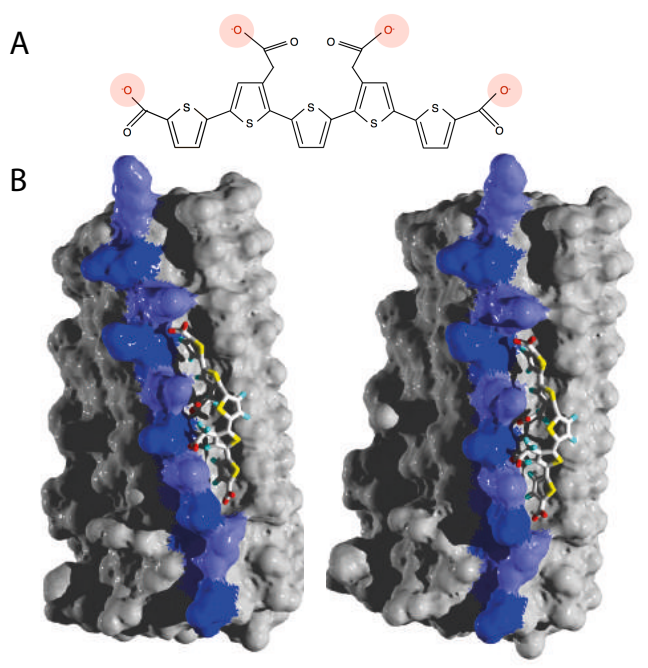


Figure 5: Binding of a luminescent polythiophene compound (LIN5001, A) by a model amyloid. (HET-s(218-289) E265K). The negative charges located on the COO groups (highlighted in red) interact with the positively charged lysines (K229, E265K) which are found in adjacent layers forming a ladder of positive charges. (B) shows two views from different angles of (HET-s(218-289) E265K) with LIN5001 bound. The knowledge of this binding mode has been used to optimize the polythiophene structure in silico to obtain more efficient anti-prion drugs. Figure adapted from \{Herrmann:2015en\}.

\section{Opening the Pandora box: Amyloid polymorphs and oligomer intermediates}

An important question remains the conformational space sampled by the different polymorphs of $A \beta$ fibrils. The finding that the two NMR structures of $A \beta 1-42$ described above characterize the same polymorph does by no mean imply that this is a canonical structure. Other NMR structures have been described \{Luhrs:2005dp\} and the cryo-EM structure of Schmidt et al.\{Schmidt:2015df\} also describes a different polymorph. Structural models for this peptide amyloid wildly differ, and range from the dimeric double horseshoe structures discussed above via single and double $\beta$-sheet turn $\beta$-sheet motifs to interlaced trimeric structures. Closely related to this structural variability are also the familial mutant forms, which again likely can each sample a certain conformational space \{Elkins:2016jj\}. Whether each mutant may sample again diverse polymorphism is not known, but in the work with the Osaka mutant polymorphism was absent, which is one reason for its being the first high-resolution $A \beta$ structure resolved.

The conformational space in an even larger context is important in the question what intermediates are formed along the pathway of fibrillization, and which are, if any, important in the context of cell toxicity and propagation of the aggregates. Oligomers formed during assembly are thought to play a central role in propagation and toxicity \{Campioni:2010hz\}. However, as stated by De Strooper and colleagues \{Benilova:2012gg\}, "This elusive soluble $A \beta$ species is in danger of becoming a way to explain inconsistencies in existing models (...)".While structural biology may shed light on these issues, the structural characterization of intermediates is sparse $\{|r i e: 2017 f|$ Kumar:2016dx\} which is attributed 
both to the lack of clarity in preparation as well as the difficulty to study them due to their time-dependent existence - an issue similar to folding intermediates in globular proteins.

Another point which remains in the dark today is the medical relevance of the fibrils studied in vitro \{Walker:2015be, MeyerLuehmann:2006hi\}. Therefore, structural studies have been performed on proteins stemming from seeded preparation using brain homogenates as seeds $\{L u: 2013 b b\}$. Different brain homogenates from different patients seem to yield a set of polymorphs for $A \beta 1-40$ that vary in their relative propensities between disease subtypes \{Qiang:2017ek\}. Still, the structural identity of the brain deposits and the seeded samples used for structural studies is not established, and structural changes could occur during the different seeding steps. For NMR, it is probably impossible to work directly on brain samples due to the need for isotope labeling $\left({ }^{13} \mathrm{C}\right.$ and $\left.{ }^{15} \mathrm{~N}\right)$ and due to the low sensitivity of the method. Possibly, cryo electron microscopy may shed light on that issue $\{$ Schmidt:2015df\} in the future and exciting structural data with tau amyloids have just been presented at the $13^{\text {th }}$ International Conference on Alzheimers's and Parkinson's Diseases (http://adpd2017.kenes.com).

\section{Concluding Remarks}

The availability of atomic-resolution structures opens the doors for a molecular-level understanding of amyloid function and the role of amyloids in disease. Markers (e.g. for imaging) can be developed as well as drugs that specifically interact with defined fibrils and regulate their growth and cell-to-cell propagation. Still the experiments are time-consuming and request in-vitro preparation of in the order of 10 milligram of fibril samples. The combination of NMR under fast sample rotation - which reduces sample amount requirements by more than an order of magnitude \{Agarwal:2014jr\} - and information from cryo electron microscopy may remove some of these limitations.

\section{Trends Box}

- Complete atomic-resolution structures of disease-related amyloid fibril have become available, in particular for amyloid $\beta$. They are based on solid-state NMR restraints combined with electron-microscopic mass-per-length data. 
- There is mounting evidence that functional amyloids play an important role in biology and medicine

- Amyloids show a higher structural complexity than previously assumed and this opens an avenue for rational drug design. Drugs need to be selective to not interfere with functional proteins in amyloid form.

- Polymorphism is an important feature of many amyloids which can now be explored in more detail. The structural homogeneity of a sample can now be established before biophysical experiments are performed.

\section{Outstanding Questions Box}

- How large is the conformational space available for the peptides and proteins forming fibrils. Is there a hierarchy of polymorphism with mayor forms distinguishing themselves in the position of the secondary structural elements \{Gath:2014ff\} and minor variations, e.g. only with a different twist of the fibrils which are formed from quasi-identical monomers \{Seuring:2017hg\}.

- What is the relevance of fibrils and fibrillar tangles in the context of amyloidrelated disease? Are they the primary cause or a consequence of the disease? Is it consequently desired to dissolve or to stabilize fibrils for therapy? Fibrillar tangles often consist of several proteins. Are they polymorphically uniform for a given protein, e.g. amyloid- $\beta$ ?

- What is the medical and therapeutic importance of molecular polymorphism: are different polymorphs responsible for different strains of the disease that distinguish themselves by different clinical symptoms and request different therapy and medication.

- What is the biological role of oligomeric aggregates either on the pathway to fibrils or off this pathway?

- It is known that fibril formation proceeds between cells following typical pathways \{Brundin:2010hz\}. Is prion-like propagation of the fibril structure between cells the relevant mechanism? Is the polymorph conserved? 
Spectroscopic Developments Box

- The progress described has been possible thanks to the spectral dispersion and sensitivity provided by high-field spectrometers (typically $\geq 800 \mathrm{MHz}$ proton frequency).

- Of further importance are efficient through space polarization transfer schemes, typically a combination of DARR\{Takegoshi:2003ux\}, MIRROR\{Anonymous:2008hr\}, CHHC\{Lange:2002up\} and PAR \{dePaepe:2008bm $\}$ as well as the protocols established with microcrystalline proteins\{Castellani:2002th, Anonymous:2008cl, Loquet:2008ti\} and early fibril studies.\{Wasmer:2008gc, vanMelckebeke:2010kz\}

- Recently, proton detection under fast MAS (>60 kHz) has shown its usefulness.\{Huber:2012dr, BarbetMassin:2014er, Bockmann:2015da\} Their use in fibrils structure determination has to be demonstrated yet. Its main advantages are lower demands on the sample amount and advantages for dynamics studies. However, the enhanced risk for rotor crashes may raise issues with biosafety for some fibrils due to the production of aerosols. \{Bousset:2015cp, Brundin:2010hz\} 


\section{Glossary}

Amyloid: protein aggregates which fold such that many copies of that protein come together and form fibrils. Historically, amyloids were defined by their reactions with dyes like Congo red or thioflavin-T. Biophysically an amyloid is defined by a core with a cross- $\beta$ spine with a $4.7 \AA$ periodicity $\{$ Westermark:2007cb\}.

amyloid- $\boldsymbol{\beta}$ : peptides of 36-43 amino acids, stemming from the cleavage of the amyloidprecursor protein, that are central in Alzheimer's disease as the main component of the amyloid plaques found in the brains of patients.

Alzheimer's disease: neurodegenerative disease that impairs memory and usually starts slowly but worsens over time.

Solid-state NMR: Solid-state NMR addresses materials which are not soluble, but which can be hydrated. All material with very long rotational correlation times are considered in this context as solid.

Polymorphism: Protein monomers can assemble into different fibrils, which are of comparable stability, and thus present energetically almost equal alternative structures. Minor variations in the environmental conditions determine which polymorph is produced in vitro.

Seeding: seeding is used to prepare homogenous samples which contain only one polymorph, by propagating the most stable fibril form through several generations of seeds. HET-s(218-289): The prion domain of the fungal prion HET-s involved in heterokaryon compatibility. It was the first amyloid to have its 3D structure determined.

Isotope labeling: Biomolecular NMR studies are generally carried out on ${ }^{13} \mathrm{C}$ and ${ }^{15} \mathrm{~N}$ labeled molecules, since these isotopes show a spin-1/2. In order to label the proteins, they are produced in the presence of isotope-labeled precursors, often ${ }^{13} \mathrm{C}$-glucose and ${ }^{15} \mathrm{~N}$ ammonium chloride.

STEM mass-per-length: scanning transmission electron microscopy can determine the molecular mass per unit length of the fibril. From this quantity, the number of monomers per 4.7 Å repeat unit can be determines

Chemical shift: The exact resonance frequency of a nuclear spin is determined by its electronic environment. The difference between the frequency of a given nuclei with the one of a reference compound (for proteins typically DSS) is called the chemical shift and is typically expressed in ppm (parts-per-million) of the resonance frequency. 
Dipolar couplings: Spins are magnetic dipoles that interact with each other through the dipolar coupling, equivalent to the interaction of two bar magnets. The dipolar coupling strength depends on the inverse $3^{\text {rd }}$ power of the internuclear distance.

PAR: A radiofrequency pulse sequence which allows the recording of spectra displaying longrange distance restraints between ${ }^{13} \mathrm{C}$ atoms.

PAIN: Following the same principles as PAR, this pulse sequence allows the measurement of distances between ${ }^{15} \mathrm{~N}$ and ${ }^{13} \mathrm{C}$ atoms.

Distance restraints: NMR structure calculation proceeds via the measurement of distances which are then used in structure calculation as restraints in order to calculate, via triangulation and dedicated programs, the 3D structure of a protein compatible with as many distances (restraints) measured as possible.

Osaka mutant: $A$ deletion mutant of $A \beta$ lacking E22. The Osaka mutant $A \beta 1-40 E 22 \triangle$ is one of the $A \beta$ familial mutants which cause early-age onset of the disease.

\section{Acknowledgements:}

This work was supported by the ETH Zurich, the Swiss National Science Foundation (Grant 200020_159707 and 200020_146757), the French ANR (ANR-12-BS08- 0013-01, ANR-14CE09-0024B).

\section{References:}

\{papers2_bibliography\} 